\title{
ChemComm
}

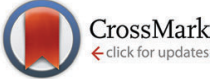

Cite this: Chem. Commun., 2015, 51, 12847

Received 4th June 2015 Accepted 6th July 2015

DOI: $10.1039 / \mathrm{c} 5 \mathrm{cc} 04610$

www.rsc.org/chemcomm

\section{A ring to rule them all: a cyclic ketene acetal comonomer controls the nitroxide-mediated polymerization of methacrylates and confers tunable degradability $\dagger$}

\author{
Vianney Delplace, ${ }^{a}$ Elise Guégain, ${ }^{a}$ Simon Harrisson, ${ }^{b}$ Didier Gigmes, ${ }^{c}$ \\ Yohann Guillaneuf ${ }^{c}$ and Julien Nicolas*a
}

\begin{abstract}
2-Methylene-4-phenyl-1,3-dioxolane (MPDL) was successfully used as a controlling comonomer in NMP with oligo(ethylene glycol) methyl ether methacrylate (MeOEGMA) to prepare well-defined and degradable PEG-based P(MeOEGMA-co-MPDL) copolymers. The level of ester group incorporation is controlled, leading to reductions in molecular weight of up to $95 \%$ on hydrolysis. Neither the polymer nor its degradation products displayed cytoxicity. The method was also successfully applied to methyl methacrylate.
\end{abstract}

Reversible deactivation radical polymerization (RDRP) enables the synthesis of well-defined, complex and functional macromolecular architectures. ${ }^{1}$ Nitroxide-mediated polymerization $(\mathrm{NMP})^{1 c}$ is perhaps the simplest RDRP technique as it does not require addition of a metal catalyst or radical initiator, but relies on reversible thermal activation of a dormant alkoxyamine end-functionality to form propagating radicals and a stable nitroxide free-radical. Although early drawbacks of NMP (e.g., requirement for high temperatures, applicability to a limited number of monomers), have been substantially overcome by the development of second-generation nitroxides such as SG1, ${ }^{2}$ the control of methacrylic esters is still a challenge. The high activationdeactivation equilibrium constant $(K)$ of SG1 results in a high concentration of propagating radicals, favoring the occurrence of irreversible termination reactions. ${ }^{3}$ The polymerization stops at low conversion and highly disperse polymers of uncontrolled molecular weight are obtained. Greatly improved control can be obtained by adding a small amount (typically $2-9 \mathrm{~mol} \%$ ) of a suitable

\footnotetext{
${ }^{a}$ Institut Galien Paris-Sud, Université Paris-Sud, UMR CNRS 8612, Faculté de Pharmacie, 5 rue Jean-Baptiste Clément, F-92296 Châtenay-Malabry cedex, France.E-mail: julien.nicolas@u-psud.fr; Web: www.twitter.com/julnicolas ${ }^{b}$ Université de Toulouse, CNRS, Laboratoire des Interactions Moléculaires et Réactivité Chimique et Photochimique UMR 5623, 118 route de Narbonne, F-31062 Toulouse, France

${ }^{c}$ Aix-Marseille Université, CNRS, Institut de Chimie Radicalaire UMR 7273, Avenue Escadrille Normandie-Niemen, F-13397 Marseille cedex 20, France $\dagger$ Electronic supplementary information (ESI) available: Experimental details, SEC chromatograms, additional kinetics, ${ }^{1} \mathrm{H}$ and ${ }^{31} \mathrm{P}$ NMR spectra, determination of the reactivity ratios. See DOI: 10.1039/c5ec04610f
}

controlling comonomer with favorable kinetic parameters (i.e., low $K$ and low cross-propagation rate constant) such as styrene (S), ${ }^{4}$ acrylonitrile (AN) ${ }^{5}$ or 9-(4-vinylbenzyl)-9H-carbazole. ${ }^{6}$ Most polymer chains were then shown to contain a unit of the controlling comonomer in the terminal position, which explained the high proportion of living chains. ${ }^{4 b}$

The flexibility and robustness offered by RDRP have led to a recent surge in the design of innovative and sophisticated vinyl monomer-based materials intended to find applications in different bio-related areas such as drug delivery or tissue engineering. ${ }^{7}$ However, because their carbon-carbon backbones resist degradation, these materials may cause prohibitive toxicity, which will hamper their translation to clinical settings and eventually to the market. On this basis, innovative strategies to confer different levels of (bio)degradability to vinyl polymers have emerged. Radical ring-opening polymerization (rROP) of cyclic ketene acetals (CKAs) is one of the most efficient strategies for the incorporation of degradable groups in the polymer backbone, enabling its complete degradation. ${ }^{8}$ Recent years have seen a resurgence of interest in CKAs, which may be explained by their ability to copolymerize with traditional vinyl monomers by both conventional free-radical polymerization and RDRP techniques. The versatility of this approach has been illustrated by the synthesis of a variety of different copolymer structures, ${ }^{9}$ which are intended for biomedical applications.

Herein, we report the discovery that 2-methylene-4-phenyl1,3-dioxolane (MPDL), a scarcely studied CKA,${ }^{10}$ both acts as a controlling monomer for the NMP of methacrylic esters and confers tunable degradability to the resulting copolymer, leading to non-cytotoxic degradation products (Scheme 1). This was applied to the SG1-mediated polymerization of methyl methacrylate (MMA) and oligo(ethylene glycol) methyl ether methacrylate (MeOEGMA); the latter being a widely-used monomer for the preparation of non-degradable, PEG-based polymers with potential biological applications. ${ }^{9 g, 11}$ The choice of NMP is particularly relevant for the preparation of polymer biomaterials as it has been shown that both SG1-terminated polymers and the SG1 nitroxide itself displayed no cytotoxicity over three different mammalian cell lines even at high doses. ${ }^{5 b}$ 


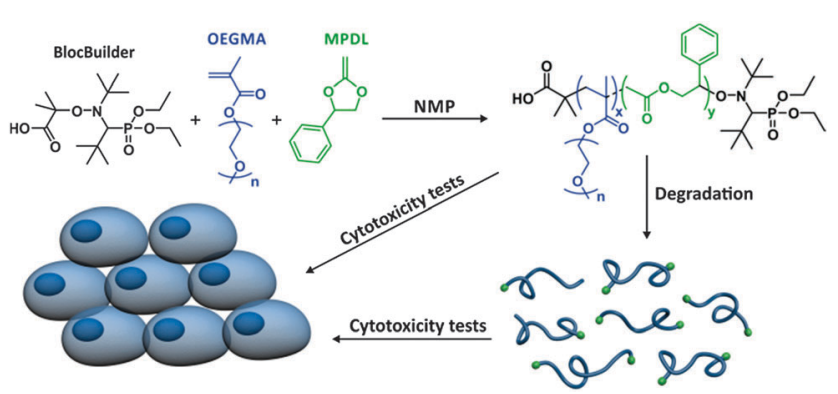

Scheme 1 Synthesis, degradation and cytotoxicity of poly[(oligo(ethylene glycol) methyl ether methacrylate)-co-(2-methylene-4-phenyl-1,3-dioxolane)] (P(MeOEGMA-Co-MPDL)) prepared by NMP.

MPDL is a 5-membered ring CKA which is easier to synthesize than the more widely used CKAs, 2-methylene-1,3-dioxepane (MDO) ${ }^{12}$ and 5,6-benzo-2-methylene-1,3-dioxepane (BMDO). ${ }^{13}$ We recently used MPDL to prepare degradable P(MeOEGMA-co-AN-co-MPDL) terpolymers. ${ }^{9,14}$ Analysis by ${ }^{31} \mathrm{P}$ NMR spectroscopy highlighted the competition between AN and MPDL to be the terminal monomer unit, with most copolymer chains terminated by MPDL-SG1 sequences. ${ }^{14}$ This set us thinking: could MPDL itself act as a controlling comonomer for the NMP of methacrylic esters and at the same time confer tunable degradability to the resulting copolymers?

MPDL was obtained after a multigram-scale synthesis (see ESI $\dagger$ and Fig. S1). The polymerization of MeOEGMA with a variable amount of MPDL $\left(f_{\mathrm{MPDL}, 0}=0-0.7\right)$ was initiated by the BlocBuilder alkoxyamine at $90{ }^{\circ} \mathrm{C}$ in $50 \mathrm{wt} \%$ toluene without additional SG1 (Fig. 1 and Table S1, expts 1-4, ESI $\dagger$ ). As expected, the homopolymerization of MeOEGMA $\left(f_{\text {MPDL }, 0}=0\right)$ was initially very fast but rapidly stopped, yielding polydisperse PMeOEGMA $\left(M_{\mathrm{n}}=12500 \mathrm{~g} \mathrm{~mol}^{-1}, D=1.72\right)$ with no control over the molecular weight. When $20 \mathrm{~mol} \%$ of MPDL was added in the comonomer feed, the situation significantly changed. Two kinetic regimes were observed. The polymerization was rapid within the first $2 \mathrm{~h}$ before slowing dramatically, likely due to the insufficient amount of MPDL units inserted to prevent irreversible termination. Some evolution of the $M_{\mathrm{n}}$ with the conversion was however noticed, matching quite well the calculated ones, with substantial improvement over the dispersities. $D$ reached 1.54 at $44 \%$ conversion for a $13300 \mathrm{~g} \mathrm{~mol}^{-1} \mathrm{P}$ (MeOEGMA-coMPDL) copolymer. At $f_{\text {MPDL }, 0}=0.4$, more rapid establishment of first order kinetics with a greater slope over $10 \mathrm{~h}$ were observed, presumably due to earlier insertion of MPDL units. This amount of MPDL was perhaps not enough to prevent the occurrence of irreversible termination reactions in the long run, as seen by the deviation from linearity at $24 \mathrm{~h}$. Nevertheless a linear evolution of $M_{\mathrm{n}}$ with monomer conversion between 7000 and $15000 \mathrm{~g} \mathrm{~mol}^{-1}$, and lower $\emptyset$ values (i.e., 1.42-1.53) were observed. A further increase in the initial amount of MPDL $\left(f_{\text {MPDL }, 0}=0.7\right)$ resulted in immediate establishment of first order kinetics up to high monomer conversion $(\sim 70 \%)$ and linear evolution of $M_{\mathrm{n}}$ over a wide range of values $\left(\sim 4400-27000 \mathrm{~g} \mathrm{~mol}^{-1}\right)$. Dispersity was also improved as it reached 1.31 at $39 \%$ monomer conversion. In fact, the higher $f_{\text {MPDL }, 0}$, the lower the dispersities during the
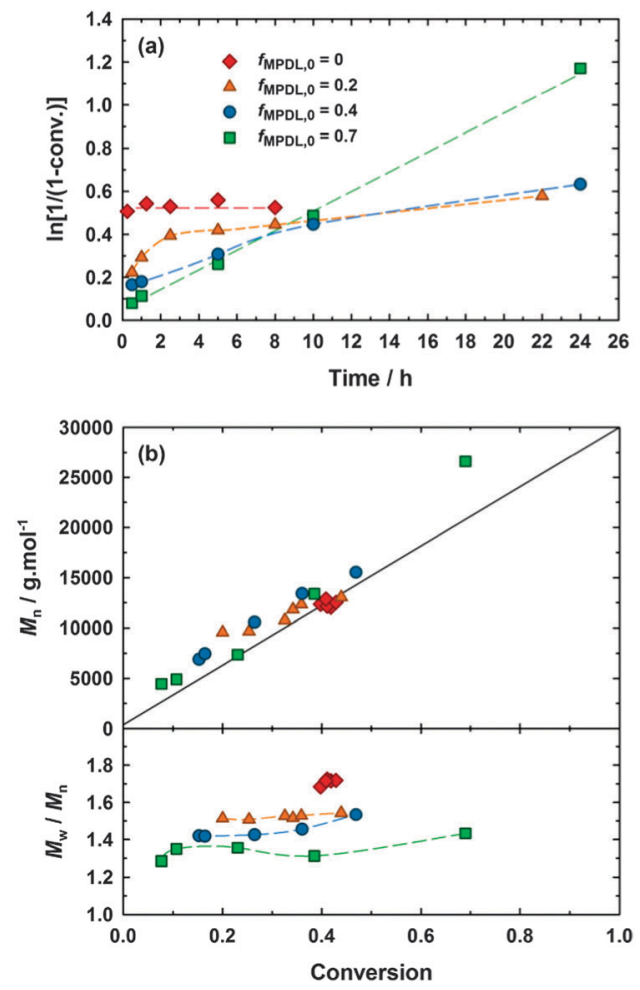

Fig. 1 NMP of MeOEGMA and MPDL in toluene initiated by the BlocBuilder alkoxyamine at $90{ }^{\circ} \mathrm{C}$, as a function of the initial amount of MPDL: ४, expt. $\mathbf{1}\left(f_{\mathrm{MPDL}, \mathrm{O}}=0\right) ; \mathbf{\Delta}$, expt. $\mathbf{2}\left(f_{\mathrm{MPDL}, \mathrm{O}}=0.2\right) ; \bullet$, expt. $\mathbf{3}\left(f_{\mathrm{MPDL}, \mathrm{O}}=0.4\right)$; 口, expt. 4 ( $\left.f_{\mathrm{MPDL}, 0}=0.7\right)$. (a) $\operatorname{Ln}[1 /(1-$ conv.)] vs. time (conv. $=$ MeOEGMA conversion). (b) Number-average molar mass, $M_{n}$, and dispersity, $M_{w} / M_{n}$, vs. conversion. The full line represents the theoretical $M_{n}$ (MeOEGMA) and the dashed ones are guides for the eye only.

polymerization (Fig. 1b). The evolution of molecular weight distribution with time further illustrated the controlled nature of the copolymerizations (Fig. S2, ESI $\dagger$ ). Two independent batches of MPDL demonstrated very good reproducibility in terms of kinetics and control, confirming the reliability of this strategy (Fig. S3, ESI $\dagger$ ). The copolymers were purified after $30 \mathrm{~h}$ by precipitation in cold diethyl ether, giving $M_{\mathrm{n}}=14700 \mathrm{~g} \mathrm{~mol}^{-1}$ and $Ð=1.55$ for expt. $2, M_{\mathrm{n}}=20100 \mathrm{~g} \mathrm{~mol}^{-1}$ and $D=1.44$ for expt. 3, and $M_{\mathrm{n}}=24500 \mathrm{~g} \mathrm{~mol}^{-1}$ and $D=1.30$ for expt. 4 .

${ }^{1} \mathrm{H}$ NMR spectroscopy of the purified P(MeOEGMA-coMPDL) copolymers (expts 2-4) showed all signals expected for a P(MeOEGMA-co-MPDL) structure (Fig. S4, ESI $\dagger$ ). The aromatic protons of the phenyl group of MPDL (signal $e$ ) were clearly visible and confirmed that its level of incorporation in the copolymer could be adjusted by varying its initial concentration in the comonomer feed. The preferential formation of the MPDL-SG1 terminal sequence was confirmed by ${ }^{31} \mathrm{P}$ NMR spectroscopy as its spectrum is very similar to that of PS-SG1 (Table S1, expt. 5, $\mathrm{ESI} \dagger)$, due to the styrene-like ring-opened structure of MPDL (Fig. S5, ESI $\dagger$ ). This analogy in structures between the open radical forms of $S$ and MPDL explains the ability of MPDL to act as a controlling comonomer. The copolymerization was also successfully performed with MMA, a representative methacrylate monomer (Table S1, 6, expt. ESI $\dagger$ ). Under similar experimental 
conditions, a well-defined P(MMA-co-MPDL) copolymer (conv. MMA $\left.(15 \mathrm{~h})=40 \%, M_{\mathrm{n}}=10200 \mathrm{~g} \mathrm{~mol}^{-1}, Ð=1.20\right)$ was prepared (Fig. S6, ESI $\dagger$ ). ${ }^{31} \mathrm{P}$ NMR spectroscopy performed in the presence of a known amount of diethyl phosphite as internal reference gave a living chain fraction as high as $85 \%$ (Fig. S7, ESI $\dagger$ ), which is similar to those obtained using $\mathrm{S}$ or $\mathrm{AN}$ as a comonomer, thus establishing the efficiency of MPDL as a controlling comonomer.

The molar fraction of MPDL in the copolymer, $F_{\text {MPDL }}$, with respect to MeOEGMA was determined by ${ }^{1} \mathrm{H}$ NMR spectroscopy to be 3.6, 11.3 and $24.8 \mathrm{~mol} \%$ for expt. $2\left(f_{\mathrm{MPDL}, 0}=0.2\right)$, expt. 3 $\left(f_{\text {MPDL }, 0}=0.4\right)$ and expt. $4\left(f_{\text {MPDL }, 0}=0.7\right)$, respectively. As for the vast majority of CKAs, unfavorable reactivity ratios impose high concentrations of MPDL in the comonomer feed in order to achieve substantial incorporation in the resulting copolymer. The reactivity ratios were determined by fitting the integrated form of the copolymer composition equation to the monomer feed composition $v s$. conversion data using the visualization of the sum of squares method, ${ }^{15}$ assuming non-negligible errors in both variables. This procedure gave a point estimate of 6.95 for $r_{\text {MeOEGMA }}$ and 0 for $r_{\text {MPDL }}$, with a 95\% joint confidence region spanning the range of 6.3-7.9 for $r_{\text {MeOEGMA }}$ and 0-0.038 for $r_{\text {MPDL }}$ (Fig. S8, ESI $\dagger$ ). The values obtained are typical for copolymerizations of methacrylate monomers with ring-opening monomers such as MDO $\left(r_{\mathrm{MMA}}=34, r_{\mathrm{MDO}}=0.057\right),{ }^{16} 1,1$-dichloro2-vinylcyclopropane $\left(r_{\mathrm{MMA}}=11, r_{\mathrm{VCP}}=0.07\right)^{17}$ and 7-methylene-2methyl-1,5-dithiacyclooctane $\left(r_{\mathrm{MMA}}=6.3, r_{\mathrm{MDTO}}=0.52\right){ }^{18}$ and reflect the lack of radical-stabilizing substituents on the double bond of the CKA.

To assess the degradability, hydrolytic degradation of the copolymers was performed in accelerated conditions (i.e., $5 \%$ $\mathrm{KOH}$ aqueous solution) and monitored over a period of $24 \mathrm{~h}$ to estimate the kinetics of hydrolysis (Fig. 2a). Adjustable incorporation of MPDL in the copolymer enabled fine tuning of the level of degradation, which typically reached its steady state after $5 \mathrm{~h}$. While the copolymer with the lowest amount of MPDL (expt. 2) led to modest degradation ( $~ 30 \%$ decrease in $M_{\mathrm{n}}$ for $F_{\mathrm{MPDL}}=0.036$ ), significant (even complete) degradation was observed for greater amounts of MPDL. When $F_{\text {MPDL }}=0.113$ (expt. 3), the decrease in $M_{\mathrm{n}}$ was $81 \%$ whereas it reached $95 \%$ for $F_{\text {MPDL }}=0.248$ (expt. 4). The complete hydrolysis of the latter was confirmed by size exclusion chromatograms which shifted toward lower molar masses (Fig. 2b). Note that the polymer without MPDL (expt. 1) was perfectly stable, thus ruling out reduction in molecular weight due to ester side chain hydrolysis.

The theoretical decrease in $M_{\mathrm{n}}$ of these copolymers can be calculated from the average sequence length of PMeOEGMA blocks in each copolymer. This can be directly estimated from the copolymer composition, as due to the very low $r_{\text {MPDL }}$, the probability of finding a sequence of two or more MPDL units is negligible. From the MPDL content of the polymers it corresponds to, on average, 1 MPDL unit for every 26.8 (expt. 2), 7.8 (expt. 3) and 3.0 (expt. 4) MeOEGMA units. These lengths correspond to $M_{\mathrm{n}}$ of 8210,2530 and $1080 \mathrm{~g} \mathrm{~mol}^{-1}$, respectively. These values are in rather good agreement with those obtained for the degraded polymers $\left(12600,3500\right.$ and $800 \mathrm{~g} \mathrm{~mol}^{-1}$, respectively), especially that with the highest amount of MPDL,
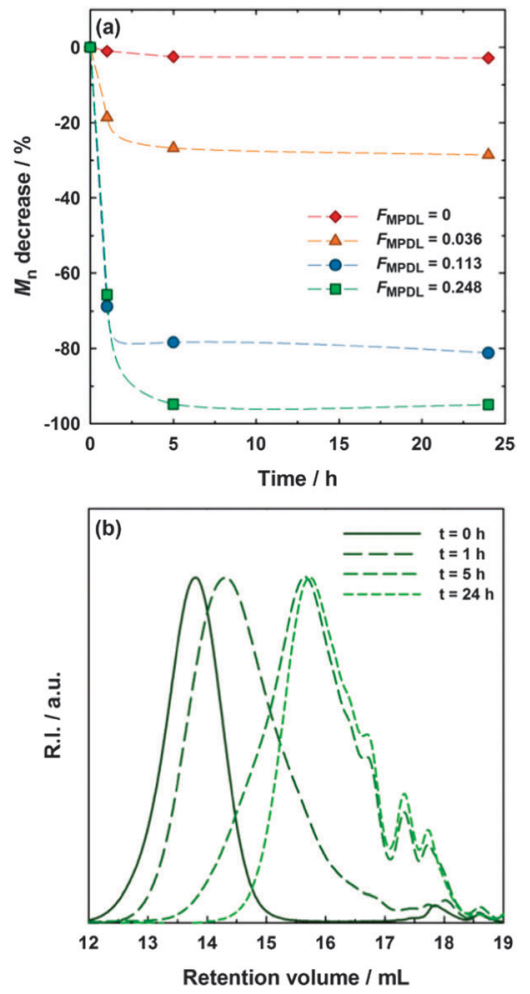

Fig. 2 Hydrolytic degradation in $5 \% \mathrm{KOH}$ of P(MeOEGMA-CO-MPDL) as a function of the content in MPDL: $\bullet$, expt. $1\left(F_{M P D L, O}=0\right)$; $\mathbf{\Lambda}$, expt. 2 $\left(F_{\mathrm{MPDL}, 0}=0.036\right) ; \bullet, \operatorname{expt.} \mathbf{3}\left(F_{\mathrm{MPDL}, 0}=0.113\right) ; \mathbf{\square}$, expt. $4\left(F_{\mathrm{MPDL}, 0}=0.248\right)$. (a) Evolution of the number-average molar mass, $M_{n}$, with time. Dashed lines are guides for the eye only. (b) Evolution of the SEC chromatograms at different time for expt. $\mathbf{4}$ during degradation.

thus confirming the near-complete hydrolysis of the mainchain ester groups.

Conferring PEG-based polymers with the ability to be degraded in a hydrolytic environment is a significant advance compared to traditional linear or comb-like PEGs that are resistant to degradation. However, neither the degradation products nor the polymer itself should be cytotoxic in an optimized product. This point is crucial as it will determine the ultimate biocompatibility of the materials. The potential toxicity of different copolymers has been tested on two representative mammalian cell types: murine fibroblasts (NIH/3T3) and murine macrophages (J774.A1). Whereas NIH/3T3 cells are one of the most commonly used fibroblast cell lines, J774.A1 cells, which play a key role in phagocytosis, were chosen to highlight possible toxicity of the copolymers after being engulfed by macrophages. The cytotoxicity was evaluated in vitro by MTT assay at two copolymer concentrations ( 0.1 and $1 \mathrm{mg} \mathrm{mL}{ }^{-1}$ ) (Fig. 3). Incubation of undegraded $\mathrm{P}\left(\right.$ MeOEGMA-co-MPDL) copolymer (expt. 3, $F_{\mathrm{MPDL}, 0}=0.113$ ) with both cell lines resulted in $>95 \%$ cell viability at $0.1 \mathrm{mg} \mathrm{mL}^{-1}$. At $1 \mathrm{mg} \mathrm{mL}{ }^{-1}$ cell viabilities were still high; 83\% with NIH/3T3 cells and $72 \%$ with J774.A1 cells. More importantly, MTT assays revealed no cytotoxicity from the degradation products whatever the cell line and the copolymer concentration. Cell viabilities were in the $97-99 \%$ range at $0.1 \mathrm{mg} \mathrm{mL}^{-1}$ and $86-92 \%$ at $1 \mathrm{mg} \mathrm{mL}^{-1}$. These results indicate the absence of obvious toxicity from the 


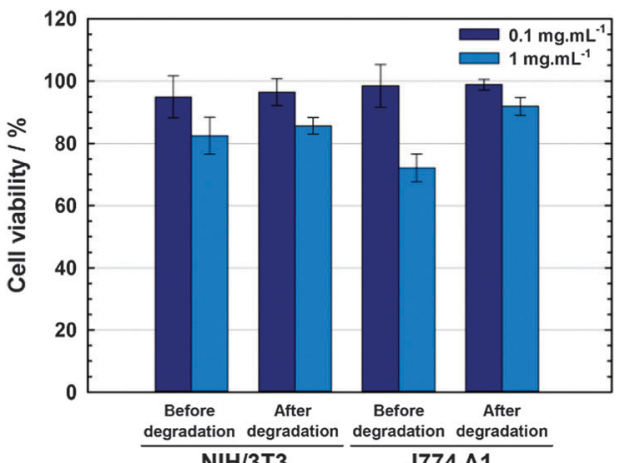

$\mathrm{NIH} / 3 \mathrm{~T} 3$

Fig. 3 Cell viability (MTT assay) after incubation of NIH/3T3 cells and J774.A1 cells with P(MeOEGMA-Co-MPDL) copolymer (expt. 3, $F_{\text {MPDL, } 0}=$ 0.113 ) at 0.1 and $1 \mathrm{mg} \mathrm{mL}^{-1}$. Results were expressed as percentages of absorption of treated cells $( \pm S D)$ in comparison to that of untreated ones as a control.

copolymer and from the products, which is a very encouraging indication of their biocompatibility and their safe use as biomaterial building blocks.

In conclusion, we have demonstrated that MPDL, a littlestudied CKA, can both act as a controlling comonomer for the SG1-mediated polymerization of methacrylic esters and confer tunable degradability to the resulting copolymer. By adjusting the comonomer feed, up to complete degradation was observed. No significant toxicity was shown, either from P(MeOEGMA-co-MPDL) or from its degradation products. Not only does this broaden the range of suitable controlling comonomers for the NMP of methacrylic esters, but it adds significant value to the copolymerization approach due to the degradability of the resulting copolymer. Additionally, this study opens up exciting perspectives in the design of new controlling comonomers and NMPderived degradable architectures with a wide range of potential (bio)applications. For instance, nanoparticles or therapeutic proteins decorated with degradable PEG segments should show reduced toxicity and persistence compared to their nondegradable PEGylated analogues.

The manuscript was written through contributions of all authors. The authors thank the French National Research Agency (ANR-11-JS08-0005) for the financial support of the PhD thesis of VD and the French Ministry of Research for the financial support of the PhD thesis of EG. Arkema is warmly acknowledged for kindly providing the BlocBuilder MA alkoxyamine and the SG1 nitroxide. CNRS is also acknowledged for financial support.

\section{Notes and references}

1 (a) M. Kamigaito, T. Ando and M. Sawamoto, Chem. Rev., 2001, 101, 3689; (b) G. Moad, E. Rizzardo and S. H. Thang, Aust. J. Chem., 2009, 62, 1402; (c) J. Nicolas, Y. Guillaneuf, C. Lefay, D. Bertin, D. Gigmes and B. Charleux, Prog. Polym. Sci., 2013, 38, 63; (d) S. Perrier and P. Takolpuckdee, J. Polym. Sci., Part A: Polym. Chem., 2005, 43, 5347.

2 (a) D. Benoit, V. Chaplinski, R. Braslau and C. J. Hawker, J. Am. Chem. Soc., 1999, 121, 3904; (b) D. Benoit, S. Grimaldi, S. Robin, J.-P. Finet, P. Tordo and Y. Gnanou, J. Am. Chem. Soc., 2000, 122, 5929.

3 C. Dire, J. Belleney, J. Nicolas, D. Bertin, S. Magnet and B. Charleux, J. Polym. Sci., Part A: Polym. Chem., 2008, 46, 6333.

4 (a) B. Charleux, J. Nicolas and O. Guerret, Macromolecules, 2005, 38, 5485; (b) J. Nicolas, C. Dire, L. Mueller, J. Belleney, B. Charleux, S. R. A. Marque, D. Bertin, S. Magnet and L. Couvreur, Macromolecules, 2006, 39, 8274.

5 (a) J. Nicolas, S. Brusseau and B. Charleux, J. Polym. Sci., Part A: Polym. Chem., 2010, 48, 34; (b) M. Chenal, S. Mura, C. Marchal, D. Gigmes, B. Charleux, E. Fattal, P. Couvreur and J. Nicolas, Macromolecules, 2010, 43, 9291.

6 (a) B. Lessard, E. J. Y. Ling, M. S. T. Morin and M. Marić, J. Polym. Sci., Part A: Polym. Chem., 2011, 49, 1033; (b) B. H. Lessard, E. J. Y. Ling and M. Marić, Macromolecules, 2012, 45, 1879; (c) B. H. Lessard, Y. Guillaneuf, M. Mathew, K. Liang, J.-L. Clement, D. Gigmes, R. A. Hutchinson and M. Marić, Macromolecules, 2013, 46, 805.

7 (a) V. Delplace, P. Couvreur and J. Nicolas, Polym. Chem., 2014, 5, 1529; (b) M. Elsabahy and K. L. Wooley, Chem. Soc. Rev., 2012, 41, 2545.

8 S. Agarwal, Polym. Chem., 2010, 1, 953.

9 (a) V. Delplace, A. Tardy, S. Harrisson, S. Mura, D. Gigmes, Y. Guillaneuf and J. Nicolas, Biomacromolecules, 2013, 14, 2837; (b) J.-F. Lutz, J. Andrieu, S. Üzgün, C. Rudolph and S. Agarwal, Macromolecules, 2007, 40, 8540; (c) J. Undin, A. Finne-Wistrand and A.-C. Albertsson, Biomacromolecules, 2013, 14, 2095; (d) J. Undin, T. Illanes, A. Finne-Wistrand and A.-C. Albertsson, Polym. Chem., 2012, 3, 1260; (e) S. Maji, M. Zheng and S. Agarwal, Macromol. Chem. Phys., 2011, 212, 2573; $(f)$ Y. Zhang, D. Chu, M. Zheng, T. Kissel and S. Agarwal, Polym. Chem., 2012, 3, 2752; $(g)$ C. Riachi, N. Schüwer and H.-A. Klok, Macromolecules, 2009, 42, 8076; (h) I. S. Chung and K. Matyjaszewski, Macromolecules, 2003, 36, 2995; (i) D. J. Siegwart, S. A. Bencherif, A. Srinivasan, J. O. Hollinger and K. Matyjaszewski, J. Biomed. Mater. Res., Part A, 2008, 87, 345; $(j)$ Y. Zhang, A. Aigner and S. Agarwal, Macromol. Biosci., 2013, 13, 1267.

10 W. J. Bailey, S. R. Wu and Z. Ni, Makromol. Chem., 1982, 183, 1913. 11 (a) B. Le Droumaguet and J. Nicolas, Polym. Chem., 2010, 1, 563; (b) J.-F. Lutz, J. Polym. Sci., Part A: Polym. Chem., 2008, 46, 3459; (c) J. Nicolas, G. Mantovani and D. M. Haddleton, Macromol. Rapid Commun., 2007, 28, 1083.

12 W. J. Bailey, Z. Ni and S. R. Wu, J. Polym. Sci., Polym. Chem. Ed., 1982, 20, 3021.

13 W. J. Bailey, Z. Ni and S. R. Wu, Macromolecules, 1982, 15, 711.

14 V. Delplace, S. Harrisson, A. Tardy, D. Gigmes, Y. Guillaneuf and J. Nicolas, Macromol. Rapid Commun., 2014, 35, 484.

15 M. Van Den Brink, A. M. Van Herk and A. L. German, J. Polym. Sci., Part A: Polym. Chem., 1999, 37, 3793.

16 G. E. Roberts, M. L. Coote, J. P. A. Heuts, L. M. Morris and T. P. Davis, Macromolecules, 1999, 32, 1332.

17 T. Takahashi, J. Polym. Sci., Part A: Polym. Chem., 1970, 8, 739.

18 S. Harrisson, T. P. Davis, R. A. Evans and E. Rizzardo, Macromolecules, $2001,34,3869$. 